\title{
Inhaltsverzeichnifs des vier und zwanzigsten Bandes, nach den Gegenständen.
}

Reine Mathematik.

Nr. der

Abandiung.

1. $\quad$ n a 1 y s i s.

1. De integratione aequationis differentialis $\left(A+A^{\prime} x+A^{\prime \prime} y\right)(x d y-y d x)$ $-\left(B+B^{\prime} x+B^{\prime \prime} y\right) d y+\left(C+C x+C^{\prime \prime} y\right) d x=0$. Auct. C. G. J. Jacobi, prof. math. ord. Regiom.

3. Demonstratio Nova Theorematis Abeliani. Auct. C. G. J. Jacobi, prof. math. ord. Aegiom. . . . , . . . . . . . . . . .

8. De integralibus differentialium algebraicorum. Aneione C. Ramus, prof. math. Hufn. . . . , . . . . . . . . . . . . .

9. Démonstration d'un thèorème sur quelques intégrales détinies. Par Mr. C. Ramus de Copenhague. . . . . . . . . . . . . . . . .

20. Démonstration d'un théorème sur les équations difŕrentielles linéaires à deux variables. Par Mr. C. Ramus de Copenhague. . . . . . .

III. 257

23. Recherches sur les formes quadratiques à coëfficients et à indéterminées complexes. Par Mr. G. Lejeune Dirichlet à Berlin. Première Partie.

III. 260

$$
\text { 2. Ge o } m e t r i e .
$$

4. Ueber die Construction der Oberflächen zweiter Ordnung, vou welchen beliebige neun Puncte gegeben sind. Vom Hrn. Dr. Hesse, Privatdocenten an der Universitât zu Königsberg. . . . . . . . . . . . .

5. Ueber das geradlinige Sechseck auf dem Hyperboloid. Vom Hrn. Dr. Hesse, Privatdocenten an der Universität zu Königsiberg. . . . . .

7. Aphorismen aus der Geometrie des Haumes. Vom Herm Prof. Dr. Plücker in Boun. . . . . . . . . . . . . . . . . . . . . . . .

22. Fortsetzung derselbets. . . . . . . . . . . . . . . . . .

10. Entwickelung eiuiger trigonometrischen. Formeln durch Hülfe der Lehre von den Doppelschnittsverhältnisseu. Vom Hrn. A. F. Möbius, Prof. in Leipzig.

11. Sur le maxinum et le minimum des figures dans le plan, sur la sphère et dans l'espace en général. Par Mr. J. Steiner, membre de l'Académie de Berlin. Premier mémoire. . . . . . . . . . . . .

16. Suite. Second mémoire. . . . . . . . . . . . . . . . . III. 189 
Nr. der

14. Note über die analytischen Beweise elementar - geometrischer Sätze. Von Herrn Dr. Reuschle, Professor am Gymnasio zu Stuttgart. . . . . . Heft. Seite.

17. Construction des regulären Siebenzehnecks. Vom Herrn Prof. v. Staudt II. 171

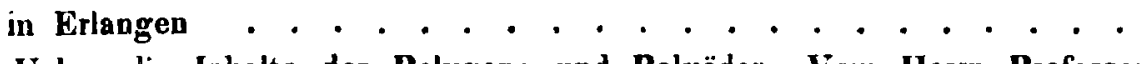

18. Teber die Inhalte der Polygone und Polyëder. Vom Herrn Professor v. Staudt in Erlaugen. • • • • . . • • • • • • . • • • • •

III. 251

21. Theorie der Centralen. Vom Herrn H. Grafsmann, Lehrer der Mathematik zu Stettin.

III. 252

24. Fortsetzung dieser Abhundlung. . . . . . . . . . . . . . . IV. 372

3. M c c h a n i k.

2. De motu puncti singularis. Auct. . G.J. Jacobi, prof math ord. Kegiom.

6. De Aequilibrii formis Ellipsoidicis. Auctore C. O. Meyer, muneris Schol. Cand., vet. Semin. Phys.-Math. Regiom. sodali. . . . . . . . . .

12. Sur le mouvement des fluides. Par Mr. A. F. Suarberg à Stockholm. (Présenté à l'Academie des sciences de Stockholm 13 Nov. 1839.) . .

I. 5

I. 44

15. Ueher das Princip der kleinsten Wirkung. (Principe de la moindre action.) Von Herm J. Zech, theol. cand. zu Tübingen. . . . . . . . . .

II. 153

II. 177

$\nabla$ e $x$ s e h i e d e n e s.

9. Sur une question de probabilité relative aux corrections des hauteurs barométriques. Par Mr. C. Ramus, prof. à Copenhague. . . . . . .

13. Probabilité des résultats moyens tirés d'observations répetées Par $M r$. Lobatschewsky, recteur de l'université de Cazan. Wac simile ciner Handschrift vou Laplact. . . . . . . . . . . . I.

- - - - - - = - Fufs. . . . . . . . . . . . II.

- - - - - - - - Legendre. . . . . . . . . . . IV.

- - - - - - - - - Huyghens. . . . . . . . . . . IV.

Druckfehler Im 17. und 20 Bande.. . . . . . . . . . . . . . . IV. 383 